selben eingedrückt wird. Es ist also ebenfalls ein Reliefgelatinebild, welches nach dem Trocknen dem Papier anhaftet, und dessen Lichtpartien durch das weisse Papier selbst dargestellt werden. Hier nehmen also die vertieften Stellen der Druckplatte die Farbe an, während dagegen bei den anderen photographischen Druckverfahren, so auch bei der Alberttypie, die erhabenen Stellen der Matrize die Drnckfarbe aufnehmen, letztere bedürfen demnach nicht erst eines Umdruckes, wie die nach Woodbury erzeugten Reliefplatten. Des letzteren Abdrücke sind, als aus Gelatine bestehend, in heissem Wasser löslich; um sie unlöslich zu machen, kann man die Bilder durch Eintanchen in Tanninlösung färben und gleichsam gerben.

Auf gleiche Weise ertheilt man auch den Gelatinereliefs, bevor sie den weiteren Manipulationen unterworfen werden, grössere Festigkeit.

\title{
Ueber die Schnellessigfabrikation und Mycoderma aceti.
}

Von Dr. C. Sommer, Apotheker zu Schwarzenfels.

Die jüngste Abhandlung unscres grossen Chemikers J. v. Liebig: Ueber Gährung, über Quelle der Muskelkraft und Ernährung, in den Annalen der Chemie und Pharmacie, 1870, hat die Mykologen zu verschiedenen Schriften, für und gegen, veranlasst.*) Die Mykologen haben Pasteur gegeniiber festgestellt: Die Behauptung, einer jeden speciellen Gährungsform liege ein specifischer, besonderer Fermentorganismus zu Grunde, sei aufzugeben und gehöre keiner der kleinen Organismen, welche hier in Frage kommen, einer einzigen Gährungsform ausschliesslich an. Das Wort Fermente oder "Hefen" sei daher im morphologischen Sinne als ein Collectivbegriff erkannt worden. **)

*) Mykologisehe Berichte von He rm. H off m a nn, Giesen 1870 u. 1871.

**) Daselbst 1871 S. 59. 
Ebenso tritt J. v. Liebig gegen die Behauptung Pasteur's, die Alkoholgährung sei ein physiologischer, die Ernährung der Hefenzelle begleitender Act, in seiner obigen Schrift auf; or weisst nach, dass der chemische Vorgang der Gährungserscheinungen auf eine chemisch-physikalische Ursache zurückzuführen sei, und lenkt die Anfmerksamkeit auf die Wirkung, welche ein Stoff im Zustande einer Molekularbewegung auf einen zweiten, hochzusammengesetzten ausüben muss, dessen Theile, durch eine schwache Anziehung zusammengehalten, in einer gewissen Spannung sich befinden.

Herm. Hoffmann bemerkt hierzu:*) „Es ist klar, dass Liebig ebensowenig, wie Pasteur, die Alkoholgährung erklärt hat, denn der Vergleich mit der Diastasewirkung ist keine Erklärung, sondern eine Constatirung einer möglicher Weise analogen Thatsache. Erklärt sind beide nicht, gewiss ist nur, dass die Hefegährung an der lebenden Hefe haftet. Der innere Vorgang, wie ihn Liebig sich denkt, ist eine Hypothese, welche vielleicht richtig ist; sie ist durch keine bessere ersetzt in ihrer dermaligen Form, aber chemisch erwiesen kann man sie schwerlich nemnen. Ausserdem leidet sie an dem Fehler allzugrosser Allgemeinheit, denn in letzter Instanz bernht alle chemische Thätigkeit, namentlich im Organismus, auf mitgetheilter Bewegung."

Bei der Essiggährung schreibt Pasteur der Essigmutter (Mycoderma aceti) eine ähnliche Thätigkeit zu, wie der Hefe und sagt: "der Essig ist das Product der Essigmutter." Dagegen behauptet Lie bi g**), dass die Essigbildung aus Spiritus nicht bedingt ist durch einen physiologischen Prozess, die Essigsäure ist nicht ein Product von Mycoderma aceti, sondern das Product eines Oxydationsprocesses.

Hierbei stiitzt sich J. v. Lie bi g besonders auf die Schnellessigfabrik von Herrn Riemerschmied in München, welcher einen Buchenholzspan aus der untersten Schicht eines Essigbilders, der ununterbrochen seit 25 Jahren nach der-

*) Mykolngiseher Berieht. 1871 S. 62.

**) Ueber Gährung ete. S. 56 . 
selben Art und Weise im Betrieb ist, vorlegt und frei von Mycoderma aceti fand. Dabei soll nichts anderes, wie verdünnter Alkohol und etwas unfertiger Essig von der vorhergehenden Operation zugesetzt werden.

Wenn die Feststellung der Species mikroscopischer Gebilde, die Art der Thätigkeit derselben in physiologischer Beziehung etc. Sache der Physiologen und Mykologen ist,. so dürte jene Angabe des Herrn Riemerschmied, sowie die Schlussfolgerung des Herrn v. Liebig, dass bei der Schnellessigfabrikation mittels Hobelspänen die Mitwirkung von Mycoderma aceti nicht nöthig sei, doch ron einem Sachverständigen bezweifelt werden.

Nach meiner langjährigen Erfahrung halte ich bei der Schnellessigfabrikation die leben de Essigmutter ebenso nöthig, wie bei der Alkoholgährung die lebende Hefe. Dass die Späne in der untern Schicht der Essigbilder ohne Mycoderma aceti gefunden werden, kann ich nur bestätigen. Das beweist aber solange Nichts, bis nachgewiesen und festgestellt ist, dass sie auch in der obersten Schicht fehlt! Zeigt Herr Riemerschmied aus seiner obern und obersten Lage Spähne, die mindestens $1 / 2$ Jahr in Anwendung und im Innern der Bilder sich befinden, ohne Mycoderma aceti, so will ich meinen $Z$ weifel fahren lassen.

Nach meinen Erfahrungen fand ich sie stots in der obern Lage; wo die Mycoderma aceti fehlte, bildete sich anch kein Essig. Die obere Schicht Späne muss bei der grössten Anfmerksamkeit und Reinlichkeit von Zeit zı Zeit erneuert werden, indem durch eindringenden Staub etc. die Thätigkeit der Mycoderma aceti verringert oder ganz aufgehoben wird.

Wer, wie ich, wiederholt Gelegenheit hatte, neue Schnellessigfabriken einzurichten und schon vorhandene aufubessern, wird wissen, dass bei genauster Beobachtung der l'emperatur, des Säure - und Alkoholgehaltes der Mischnng u. s. w. dennoch nach circa 1/4 Jahr die Essigsäurebildung stockt, um schliesslich ganz einzuschlafen. Hat man die Essigfabrik für Fremde eingerichtet, so kommt man in den Verdacht, mehr versprochen zu haben, als man halten kann, wird wohl gar 
für einen Schwindler gehalten. Was ist das? Es werden alle auftreibbaren Schriften über Schnellessigfabrikation nachgeschlagen, alle möglichen Geheimmittel, Zucker, Weinstein, Sanerteig a. s. w. zugesetzt. Doch kein Buch giebt Auskunft darüber. Am hesten ist, man wartet nun $a b$, bis sich Mycoderma aceti grebildet hat. Dicess bewirkt man durch einen sehr geringen Zusatz von Bier oder Weinmost, das heisst, durch Stoffe, welche die Bildung der Mycoderma aceti befördern. Hat man Essigmutter auf seinen Spänen, dann muss die Zumisehung so gehallon werden, dass dieselbe nicht überhand nehmen kann und, die Quelle stets sich wiederholender Störungen" "wird, wie J. v. Liebig S. 51 seiner Schrift ganz richtig bemerkt.

Ferner war es mir bis jetzt ganz unmöglich und viele andere Fabrikanten, welche ich darüber gesprochen, sind derselhen Ansicht, mit reinem verdünnten Weingeist und unfertigem Essig der vorhergehenden Operation, Essig zu erzengen. Stets fand ich, dass jeder Fabrikant offen oder im Gcheimen, solche Zusätze machte, die ihm oft für hohe Summen verrathen wurden und wolke er am geeignetsten hielt.

Wiedorholt habe ich mitten in der besten Essigbildung: :hle Zusätze weggelassen, nur verdünnten Weingeist und Essig von friher augesetat und wie gewöhnlieh weitergearbeitet. lis ging einigo Zeit gnt, so lange die Essigmutter noch Billungsstoffe vorfand; waren diese aber erschöpt und die Mycoderma aceti ausgclaugt, so wurde der Essig immer schwächer, bis die Bildung desselben ganz aufhörte. Setzte ich hieranf etwas Bier oder dergl. hinzu, dann begann allmählig wieder ein Steigen des Essigsäuregehaltes.

Anderseits muss es jedem Fabrikanten und dem, wel(her sich mit Interesse dem Studium der Essigbildung zuwendet, aufgefallen sein, daws bei der Schnellessigfabrikation nur ein Essig (sogenannter Essigsprit) erzeugt werden kann ron einem Gehalte nicht über $9 \%$ wasserfreier Essigsäure!

Hat man 4 Essigbilder (gewöhnlich sind es nur 8), welche zusammon arbeiten, wobei in regelmässigen $\mathrm{Zwischen-}$ 
räumen von einem zum andern aufgeschüttet wird, so wird man bemerken, dass der 4 te Bilder mit dem höchsten Essigsäuregehalte, wärmer gehalten und zeitweilig mit dem 1 ten oder 2 ten Bilder gewechselt werden muss. Er besitzt immer in Innern die niedrigste Temperatur von allen 4 Bildern, weil eben die Oxydation des Weingeists bei ihm am langsamsten erfolgt. In einem Essig von über $9 \%$ Essigsïure fand ich keine bildungsfähige Mycoderma mehr vor. Stellt man gut abgewaschene Mycoderma aceti in einem Gemische von reiner 10 proc. Essigsüure und $2 \%$ Alkohol bei einer Temperatur von $16^{\circ} \mathrm{C}$. einige Tage bei Seite, so findet keine Vermehrung derselben statt. Sie ist anch nach eirigen Tagen nicht mehr im Stande, gehörig ausgelangt, in einem normalen Gemische von verdïnntem Weingeist und 3 proc. Essig sich zu vermehren oder den Essigsäuregehalt zu erhöhen.

Wäre die Essigsäurebildung aus Weingeist nur ein reiner Oxydationsprozess, unabhängig von Mycoderma aceti, so müsste unthwendig bei vermehrtem Weingeistzisatze in den Essigbildern der Essigsäuregehalt steigen. Fin vermehrter Zusatz von Weingeist zu einem Rssigsprit von 8 bis $9 \%$ Essigsänre bedingt aber nur einen grössern Gehalt an Weingeist im ablaufenden, fertigen Essig, was natiirlich 7,1 grossen Verlusten Veranlassung giebt.

Am ippigsten wuchert die Essigmutter nach dem Zusatze von etwas Bier gu einem schwachen Essig von 2 bis $3 \%$ Essigsäure und ebensoviel Weingeist. In den Essigbildern, welche einen stärkeren Essig produciren, hat man von einer Ueberwucherung des Pilzes wenig zu befürchten. Bei den Bildern mit schwachem Essigsäuregehalte muss man mit der Zugabe von Bier vorsichtig sein, damit durch die vermehrte Bildung des Pilzes die Luftcirculation im Innern nicht gehemmt wird. Am leichtesten gelingt es bei der Schnellessigfabrikation, mit 3 Bildern einen Essig mit $6 \%$ wasserfreier Esigsäure zu erzielen. In der That fand ich selten ein Product von höherem Gehalte. Die Schnellessigfabrikation ist die rationelle Cultur der Mycoderma aceti. 
„Es ist ganz unbezweifelbar, sagt J. v. Li e big, die Essigmutter die Oxydation des Weingeists zu Essin zu repmitteln vermag, aber diese Wirkung beruht nicht anf einem physiologischen Vorgange. Der Weingeist bedarf zu seinem Uebergange in Essigsäure nur Sauerstoff, den ihm die Mycoderma aceti aus ihrer Substanz heraus nicht geben kann und nicht giebt. Die Analyse der Luft, welche die Essigbilder verlässt, beweist, dass der zur Oxydation des Weingeists dienende Sauerstof von der Luft genommen wird, und der einzige Antheil, den die Essigmutter an diesem Processe nimmt, kann nur darin bestehen, dass durch sie diese Aufnahme vermittelt wird; sie ist nur durch diese chemische Eigenschaft wirksam und kann als lebende Planze, durch eine ganze Anzahl todter Stoffe nndPflanzentheile vertreten werden. " Hier ist die Vertretung der Mycoderma aceti dureh Platinmohr, Hobelspäne, Birkenreiser, Traubentrester, Kohle etc. in's Auge gefasst. Von allen diesen Stoffen findet bei uns nur die Anwendung von Hobelspänen Beifitl. - Holzkohle fand ich ganz unbrauchbar.

Wie ich schon oben erwähnte, hört zum Verdrusse des Besitzers bei neu errichteten Essigbildern nach einiger Zeit die Essigerzeugung auf und beginnt erst wieder mit der Entstehung der Essigmutter.

Wir haben hier offenbar 2 Arten von Essigbildung. Zuerst mag allerdings die Bildung vor sich gehen, wie beim Platinmohr durch Verdichten oder Ozonisiren des Sanerstoffes, Oxydiren des Weingeists zu Aldehyd und Umwandlung des letztern in Essigsäure.

Nach einiger Zeit ruht aber die Essigbildung beim Plafinmohr ebenso gut, wie bei den Hobelspänen; die Fabrikation steht still, gerade wie oben angegeben.

Ob sich dabei im Platinmohr ähnliche Zersetzungs - und Oxydationsproducte bilden, wie bei der Anwendung des Platinschwammes zu der Döbereiner'schen Zündmaschine, würde noch festzustellen sein.

Ueber Gährung etc. S. 55, n. 50. 
Unzweifelhal't ist die Thätigkeit des Platinmohrs eine mindere, als die der Mycoderma aceti. Nach J. W. Döbereiner's Angaben:) soll aus 33\% Weingeist ein so starker Essig erhalten werden, dass er mit dem 8 bis 10 fachen Gewichte Wasser verdïnnt werden muss, um zum gewöhnlichen Gebrauche zu dienen. Nehmen wir nur eine Stärke von $3 \frac{1}{2} \%$ Essigsänre in dem verdünnten Essig an, so muss das erhaltene Product mindestens 28 bis $30 \%$ Essigsäure enthalten haben. In einer so starken Sïure schrumpfen sowohl die Zellen der Mycoderma zusammen, als auch die Essigälchen (Vibrio aceti) zerstört werden. Lebende Organismen sind darin unwöglich. - Wie oben gesagt, erzengt aber Mycoderma aceti nie einen Essig von einem höheren Säuregehalte, wie 9\%. Nachdem der in den Poren der Hobelspäne verdichtete und ozonisirte Sauerstoff verbraucht ist, tritt zweitens die Mycoderna aceti an die Stelle und leitet die Oxydation des Weingeists ein. Ias durch die Späne laufende Essiggut wird durch die von unten konmende Luft weiter oxydirt. Man findet in der obern Lage der Späne die höchste Temperatur des Essigbilders, je weiter nach unten, desto niedriger ist dieselbe.

Bei best geleiteter Bildung von Essig findet man in dem Producte stets noch Aldehyd und Weingeist; je wenigor ron beiden noch darin ist, desto höher der Essigsiiuregehalt, um so vortheilhafter für den Fabrikanten und um so vollkommener ist die Darstellungsweise.

In den chemischen Werken wird der schönen Entdeckung F. W. Döbereiner's, die Erzengung von Essigsäure mittols Platinmohr, nur ein theoretisches Interesse zugetheilt. Doch wäre es einos Preises würdig, festzustellen, welche Hindernisse der Austentung dieser Bntdeckung im Grossen entgegen stehen.

Ende December 1870, nachdem ich in der Pharmaceutisehen Centralhalle Nr. 50 unter der offenen Correspondenz

*) Luehrbuch der praktischen $u$, theoretischeu Pharmacie $\mathbf{~}$. Dr. $F^{\text {. }}$ Döbereiner, 3. Theil, S. 454 . 
Dr. Hager's Ansichten über Pasteur's Entdeckung, er neant sie Phantasien, gelesen hatte, theilte ich demselben kurz meine Erfahrungen mit, bat ihn, das Nöthige zu veroffentlichen und mir Werke anzugeben oder zu übermitteln, in welchen Ausführlicheres über die Darstellnng der Essigsäure mittels Platinmohr enthalten sei.

Ich beabsichtigte weitere Versuche darüber anzustellen. Hierauf theilte mir Dr. Hager mit, dass er schon vor 25 Jahren Versuche mit Platinmohr gemacht habe. Das Verfahren habe nur ein theoretisehes Interesse, der Platinmohr werde bald unthälig u. s. w. Vielleicht findet sich Mancher dutch vorstehende Mittheilung veranlasst, diese Frage wieder aufzunehmen, oder gelehrte Körperschaften $u$. d. gl. stellen eine Summe dazu zur Verfügung. Die Hauptansgabe vernrsacht der Platininohr; dieser findet nach beendigten Versuchen immer wieder Verwerthung.

Inzwischen haben die Pasteur'schen "Phantasien" doch einen reellen Erfolg erzielt, wenn man den französischen Berichten Glauben schenken darf. Nach Angabe der dentsehen Industrie-Zeitung $\mathrm{Nr} .24$, 1871, lat Breton-Langier in Orleans, indem er das von Pasteur im Jahre 1862*) vorgeschlagene Verfahren, Essig aus W ein ohne weitere Hülfsmittel als Iyycoderma acoti zu bereiten, in Grossen austührte, den Preis von $1000 \mathrm{Fr}$. fü Verbesserung der Essighabrikation von der Pariser Societé d' Encouragement erhalten.

Welche Stelle die Mycoderma aceti bei der Schnellessig. fabrikation, besonders wem man durch blosse theoretische Speculation geleitet die praktischen Erfuhrungen unbericksichtigt lïsst, spielt, will ich noch an folgenden Erlebuissen zeigen.

Vor ungefähr 10 Jahren hatte ich Gelegenheit, in einer chemischen Fabrik, nicht weit vom Rhein gelegen, eine Anstellung zu finden. In dieser Fabrik wurden ansser ver-

*) Dingler's Polytechnisches Journal Bd. CLXV S. 303. 
schiedenen pharmaceutisch - chemischen Producten, anch in 30 Essigbildern von 12 Fuss Höhe und 4 Fuss Durchmesser. eine enorme Menge Essig dargestellt. Leider war die Essigfabrikation schon vor meiner Ankunft den Händen zweier träger Arbeiter überlassen worden. Diese schiutteten das meist triibe Gemisch von Bier oder Wein noch sehr unregelmässig auf u. s. w. Es blieb dann auch nicht aus, dass die Luftzinge durch die Hobelspäne theils durch mechanische Verunreinigungen, theils durch Massenanhäufung von Mycoderma aceti verstopft wurden. Der Essig wurde langsam, aber stetig schwächer. Die Luft in dem Essiglocale wurde so mit Aldelhyd gefüllt, dass der eine Arbeiter wiederholt Angenentriindung bekam; ein sicheres Zeichen einer unvollständigen Oxydation, einer gehemmten Luftcirculation in Innern der Essigbilder. Man schritt zum Entleeren und Erneuern der Bilder. Die Hobelspäne waren in der oberen Schicht ausser mit Schmutz, meist mit ganzen Klumpen Mycoderma aceti verunreinigt. Weiter abwärts fanden sich Knäuel von verrotteten, mit weissem Schimmelpelz überzogenen Spänen. Die Späne selbst waren mehr oder weniger schwarz, zu Pulver zerreibbar. In der Nähe der unteren Luftlöcher fanden sich ganze Hände voll Maden der Essigfliege.

Inzwischen hatte sich ein Mann in den Zeitungen angeboten, ein neues Verfahren zu lehren, Schnellessig darzustellen, wobei das stiindliche Aufschütten nicht erfordert würde u. s. w. Die Chefs der Fabrik kauften sein Geheimniss, der Mann selbst richtete die Essigfabrik ein. Nach dessen Principe sollten des Morgens 24 Fässer, welche sich über den Essigbildern befanden, mit Essiggut vollgepumpt werden. Mittels vines Krahnes und einer sinnreichen Tröpfelvorrichtung sollte ver Inhalt dieser Fässer innerhalb 24 Stunden über die Iobelspäne in den Bildern vertheilt werden. Der starke Essig wurde entfernt und der schwache alsdann wieder in die Höhe gepumpt. Bei dieser genialen Einrichtung war nur das das Merkwürdige, nach der Aussage eines meiner Chefs (ein berühmter Mann von sehr bekanntem Namen), dass man nicht schon früher selbst auf diese Idee gekommen war. Nach circa 
4 Monaten war die Umwandlung der Essigfabrjk vollendet; ich konnte ror Ungeduld die Zeit kam erwarten, wann das grosse Pumpwerk in Gang gesetzt worden sollte.

Die Essigbilder wurden wie gewöhnlich angesäuert und zum Essiggute nichts verwandt, wie verdünnter Weingeist und Essig von früherer Operation, und als dieser nicht ausreichte, destillirter Essig von einer renommirten Sehnellessigfabrik in Würtemberg. "Doch grau ist alle Theorie etc." Es ging nicht. Zuerst platzte überall die 2 Zoll weite, mit 1/4 Zoll dicker Wand versehene Zinnröhre; sie konnte dem Drucke der 19 Fuss hohen Essiggutsäule, selbst dann nicht widerstehen, nachden sie in eine Fichtenholzröhre eingeklemmt war. Es wurde nun zu einer Holzpumpe gegriffen, doch anch diese erwies sich unbrauchbar; sie wurde fortwährend undicht. Man musste das Essiggut wieder durch mehre Männer in die Hohte tragen lassen. Dio Vertheilnng auf den Hobelspänen erfolgte nun granz herrlich; es gab aber keinen Essig!

Zu derselben Zeit wurde $P$ asteur's Cultur der Mycoderma aceti bekannt; ich schlug nach einigen Wochen vor, die Salzlösungen der Phosphate etc. zusetzen, um Mycoderma auf die späne zu bekommen. Dieses geschah in dem von Pasteur angegebenen Verhältnisse. Nach 5 bis 6 Tagen, (es war Anfangs Mai) hatte sich eine solche Menge Mycoderma aceti gebildet, dass sie die ganze Tröpfelvorrichtung verstopfte und an jedem Morgen 3 bis 4 Zoll lange Fäden derselben an den Oeffnungen der Krahne hingen. Man musste den ganzen Tag dazu verwenden, die Oeffnungen wieder aufzuräumen. Obwohl der Sänregehalt im Grunde des Fissigbilders um einige Proc. zugcnommen hatte, so wurde doch nach kurzer Zeit die ganze Vorrichtung', welche über 1000 Thaler gekostet hatte, bei Seite geworfen und zur alten bewährten Methode zurück gegriffen. Würde man ohne Mycoderma arbeiten können, so würde die besprochene Methode mancherlei Vorzüge vor der früheren haben. Aber selbst nach einem sehr geringen Zusatze der Phosphate etc. wurden dennoch alle' Tröpfelvorrichtungen durch Mycoderma aceti verstopft. 
Ein ähnliches Loos traf ein um eine senkrechte Axe bewegliches Krenz, dessen Arme hohl und mit einer Tröpfelvorrichtung versehen waren, das der Besitzer in Höchst an der Weser für 10 Thaler ans Braunschweig geholt hatte!

Dasselbe Resultat lässt sich bei dem W. S te g er's EssigGenerator nach Dr. W. Reinemann in Berlin*) erwarten. Es werden gar bald die vielen kleinen Oeffnungen ihren Dienst versagen.

Meine Versuche über Mycoderma aceti werde ich fortsetzen, ebenso iiber Vibrio aceti, und werde ich später suf die Verwerthung der mit Aldehyd und Essigsäure beladenen Luft zurückkommen.

\section{Gewinnung metallischen Antimons.}

Nach R. F. Smith, Glasgow, werden die feingepulverten Antimonerze in heisse Salzsäure (in hölzernen Trögen) eingetragen, die Chlorantimonlösung wird abgezogen und in dieselbe Zink oder Eisen eingefiihrt. Man wäscht und trocknet den Niederschlag und schmilgt ihn in Tiegeln unter einer Kohlenstaubdecke zusammen. (Specif. $v$. Patenten

Grossbrittannion u. Irland; d. 26. 2. 71; Ber. d. deutsch. chem. Gesellsch. Berlin 13. Nov. 1871, S. 855.).

H. $L$.

*) Industrie-Blätter Nr. 11, 1869. 